\title{
INVESTIGATION OF A TYPHOID NOTIFICATION
}

A case of typhoid fever was notified to the Public Health Unit of the Northern Sydney Area Health Service on March 18, 1991. The patient was an 11-month-old boy in the children's ward of Hornsby Ku-ring-gai Hospital. He had been in India from October 1990 and had not had any illness while there. His mother was first ill on February 13, 1991 and was admitted to hospital in India. She was diagnosed as having typhoid fever and treated with intravenous antibiotics. Mother and child returned to Australia on March 4.

On March 7 the boy became febrile and saw his GP, who prescribed Amoxicillin and Paracetamol. By March 9 he was still febrile and was prescribed Trimethoprim/ Sulphamethoxazole in place of Amoxicillin. On March 10 he had diarrhoea three times after his second dose of
Trimethoprim/Sulphamethoxizole, and still had a high fever. The GP advised referral to Accident and Emergency at the Hornsby Ku-ring-gai Hospital. He was not febrile while there and was sent home on Amoxicillin after a diagnosis of Otitis Media. On March 12 he was still febrile and the GP arranged for his admission to hospital.

He was intermittently febrile and drowsy after admission, but at other times was cheerful and playing normally. His bowels were normal, he was eating and drinking normally and he had no cough. There were no spots on the trunk and there was no bradycardia. There was no splenomegaly and no lymphadenopathy.

The baby's white cell count was 14.8 , AST was $70(0-41)$ \& ALT 81 (0-65). Stool and blood cultures were taken. Giardia was seen in his stool on March 13 and he was

\section{FACT FILE + FACT FILE + FACT FILE + FACT FILE + FACT FILE + FACT}

\section{IDENTIFICATION}

Typhoid fever is a systemic bacterial disease characterised by insidious onset of sustained fever, headache, malaise, anorexia, a relative bradycardia, splenomegaly, rose spots on the trunk, non-productive cough, constipation more commonly than diarrhoea (in adults) and involvement of the lymphoid tissues.

The usual fatality rate of 10 per cent can be reduced to less than 1 per cent with prompt antibiotic therapy. Relapses occur in 5-10 per cent of untreated cases and may be more common (15-20 per cent) after antibiotic therapy. Mild and unapparent illnesses occur, especially in endemic areas.

The aetiological organisms can be isolated from the blood early in the disease and from urine and faeces after the first week. A four-fold rise in agglutination titre in paired sera appears during the second week in fewer than 70 per cent of cases of typhoid fever. When it occurs it supports the diagnosis, provided vaccine had not been given recently. Because of its limited sensitivity, serology is of little diagnostic value.

\section{INFECTIOUS AGENT}

Salmonella typhi, the typhoid bacillus.

Presently 106 types can be distinguished by phage typing, which is of value in epidemiological studies.

\section{OCCURRENCE}

Worldwide. Strains resistant to recommended antibiotics have appeared in several areas of the world. Multiresistant strains have been reported from Asia, the Middle East and Latin America.

\section{RESERVOIR}

Man. Family contacts may be transient carriers. The chronic carrier state is most common among persons infected during middle age, especially females; carriers frequently have gall bladder pathology.

\section{MODE OF TRANSMISSION}

By food and water contaminated by faeces and urine of patients and carriers. Important vehicles in some parts of the world include shellfish taken from sewagecontaminated beds, raw fruit, vegetables contaminated by nightsoil, contaminated milk and milk products (usually by hands of carriers) and missed cases.

\section{INCUBATION PERIOD}

The incubation period depends on the size of the infecting dose; usual range is one to three weeks.

\section{PERIOD OF COMMUNICABILITY}

As long as the bacilli appear in excreta, usually from the first week throughout the convalescence; variable thereafter. About 10 per cent of untreated typhoid fever patients will discharge bacilli for three months after onset of symptoms, and 2-5 per cent become permanent carriers.

\section{SUSCEPTIBILITY}

Susceptibility is general and is increased in individuals with gastric achlorhydria. Relative specific immunity follows recovery from clinical disease, unapparent infection and active immunisation, but is inadequate to protect against ingestion of large numbers of organisms.

\section{PREVENTATIVE MEASURES}

1. Educate the public about hand-washing.

2. Dispose of human faeces in a sanitary manner.

3. Protect, purify and chlorinate public water supplies.

4. Use scrupulous cleanliness in food preparation and handling

5. Pasteurise or boil all milk.

6. Exclude carriers from handling food and from providing patient care.

7. Immunise before entering endemic areas. 
started on Metronidazole. By March 15 he was still febrile, and so remained in hospital.

On March 16 the blood culture grew salmonella typhi which was resistant to Cotrimoxazole, Ampicillin, Choramphenicol and Tetracycline, and sensitive to Cefotaxime. Typhoid serology was Poly O (weakly positive) and Vi (strongly positive). The laboratory Microbiologist was consulted and intravenous Cefotaxime was commenced.

By March 18 the boy was afebrile and has remained well since. The Public Health Unit was notified that day. On March 22 the Cefotaxime was ceased and the baby was discharged on oral Amoxicillin/Clavulanic Acid for one week.

\section{PUBLIC HEALTH INTERVENTION}

On the day it was notified, the Northern Sydney Area Health Service PHU discussed the case with the Microbiologist, the Health Department Infectious Diseases Epidemiologist, the General Practitioner and the Hospital Infection Control Nurse Consultant. PHU staff took a full history from the patient's mother. After consultation with the GP, infection control measures such as strict hand-washing procedures and the mother not preparing any food or drink as she had recently had typhoid fever - were instituted at their home.

The only contacts in Australia were the grandmother, grandfather, uncle and mother. They were not involved with public food preparation or handling. Stool culture included all family members. Three stool samples on three different days were taken for culture looking for typhoid. All the stool samples were negative.

In follow-up all the family, including the patient, remained well. There have been no further cases of typhoid fever in the Northern Sydney Area.

\section{DISCUSSION}

This is a case of typhoid fever in an unimmunised 11-month-old child, acquired in India and diagnosed and treated in Australia soon after his return. As he had acquired the disease overseas, the Public Health investigation was limited to the immediate family.

History provided by the mother indicated that home conditions in India were good, and included running water, sewerage and refrigeration. Hypothetical sources of the typhoid could have included:

\begin{tabular}{l|l} 
& $\begin{array}{l}\text { water drunk on the train to New Delhi on the } \\
\text { journey home }\end{array}$ \\
water or food from visits near the home near \\
Bombay \\
transfer of the disease from the mother
\end{tabular}

David Pakchung, Public Health Medicine Registrar Donald Holt, Director

Public Health Unit, Northern Sydney Area Health Service

Benenson A et al. Fifteenth Edition 1990, Control of Communicable Diseases in Man, American Public Health Association, Washington.

\section{Troining for a healthier future}

\section{Continued from page 74}

Placements have also been offered at the National Health and Medical Research Council's Clinical Trials Centre, the Centre for Clinical Epidemiology and Biostatistics at the University of Newcastle, the Communicable Disease Section of the Commonwealth Department of Community Services and Health, the National Centre in HIV Epidemiology and Clinical Research and the Australian Institute of Health.

PHOs undertake, under supervision, day-to-day duties of the unit to which they are attached. At the beginning of each assignment PHOs and supervisors outline a plan of projects to be completed during the attachment.

Monthly seminars in the areas of reproductive and child health, infectious disease, chronic disease and injury prevention, environmental health and health services evaluation are held at the Public Health Division in North Sydney. PHOs are encouraged to participate in short courses on epidemiological methods, health economics, management and computer skills.

The program provides the practical experience required to join the Australian Faculty of Public Health Medicine. For non-medical PHOs it will aim to provide training for accreditation by relevant professional bodies as these develop.

Overseas training opportunities are being established with the US Centers for Disease Control (Atlanta) and the British Communicable Disease Surveillance Centre (Colindale). One PHO now participates in the US Centers for Disease Control's Epidemic Intelligence Service.

Several key experiences have been identified for PHOs to complete to meet the objectives of the program. They are:

- investigation of at least two disease

clusters/outbreaks

- substantial involvement in the establishment or evaluation of a surveillance system

- evaluation of the burden of one or more illnesses or injury types in a specific community

- substantial involvement in planning, implementation or evaluation of a public health program

- research and report on a public health problem, such as assessment of an environmental hazard publication of at least one scientific article in a refereed journal and at least three articles in the Public Health Bulletin

- presentation of a paper/poster at a scientific meeting and at least one paper/poster at the annual Public Health Officer Program Scientific Conference

George Rubin, Michael Frommer, Mark Bek, Susan Furber, Peter Lewis

Epidemiology and Health Services Evaluation Branch NSW Health Department

1. Leeder S, Rubin G. Improved public health. NSW Public Health Bulletin, $1990 ; 1: 2 \& 6$

2. Rubin G, Frommer M, Morey S, Leeder S. On the right track, NSW Public Health Bulletin, 1990; 2: 1-2

3. Ward M, King L, Rubin G, Stewart G. Setting a new agenda. NSW Public Health Bulletin, 1990; 2: 5-6. 